\title{
Posterior pole retinotomy for treatment of recurrent macular hole retinal detachment in highly myopic eyes: a pilot study
}

\author{
Xianggui Wang ${ }^{1,2}$, Xuezhi Zhou ${ }^{1,2}$, Ying Zhu ${ }^{1,2}$ and Huizhuo $\mathrm{Xu}^{1,2^{*}}$
}

\begin{abstract}
Background: To investigate the feasibility and efficacy of posterior pole retinotomy to treat recurrent macular hole retinal detachment (MHRD) in highly myopic patients.

Methods: We performed a retrospective study and reviewed the medical records in our hospital between January 1, 2016 and December 31, 2018. Highly myopic patients who received posterior pole retinotomy with silicone oil tamponade for their recurrent MHRD after pars plana vitrectomy were included in the analysis. Postoperative retinal reattachment, best-corrected visual acuity (BCVA), macular hole closure, and complications were evaluated.

Results: There were 11 patients (11 eyes) included in this study. All retinas were reattached. Silicone oil was successfully removed from all eyes 1.5-3 months after the surgery. Macular holes were completely closed in three eyes and remained flat open in eight eyes. The BCVA of all eyes improved significantly at 12 months after surgery (logarithm of the minimal angle of resolution, pre vs. postoperatively, $1.87 \pm 0.44$ vs. $1.15 \pm 0.24, P<0.05$ ). None of the patients had complications such as endophthalmitis, fundus hemorrhage, retinal redetachment, and proliferative vitreoretinopathy.
\end{abstract}

Conclusion: Posterior pole retinotomy is a safe and effective surgery to treat recurrent MHRD after pars plana vitrectomy in highly myopic patients.

Keywords: Macular hole retinal detachment, High myopia, Posterior pole retinotomy, Recurrent retinal detachment, Vitrectomy

\section{Background}

One of the most serious complications in patients with high myopia is macular hole retinal detachment (MHRD), which can result in partial or complete vision loss [1]. Currently, pars plana vitrectomy (PPV) is the primary surgical choice to reattach the retina and close the macular hole in order to restore visual function. However, due to the long axial length, posterior scleral staphyloma, and severe chorioretinal atrophy, the

\footnotetext{
* Correspondence: xhz1030@csu.edu.cn

${ }^{1}$ Eye Center of Xiangya Hospital, Central South University, No. 87 Xiangya Road, KaiFu District, Changsha 410008, Hunan, China

${ }^{2}$ Hunan Key Laboratory of Ophthalmology, No. 87 Xiangya Road, KaiFu

District, Changsha 410008, Hunan, China
}

outcome of PPV is not satisfactory even with posterior vitreous cortex clearing, epiretinal membrane and internal limiting membrane (ILM) peeling, and intravitreal gas or silicone oil tamponade [2]. It has been suggested that vitrectomy combined with macular buckling (L-type titanium plate, Ando plombe), scleral imbrications, or posterior scleral reinforcement could effectively shorten the ocular axis, alleviate posterior scleral staphyloma, and achieve retinal reattachment and macular hole closure [3-6]. However, these surgical procedures are complicated and can have certain adverse outcomes. Therefore, it is important to find new treatment options for myopic patients with recurrent MHRD after PPV.

(c) The Author(s). 2021 Open Access This article is licensed under a Creative Commons Attribution 4.0 International License, which permits use, sharing, adaptation, distribution and reproduction in any medium or format, as long as you give appropriate credit to the original author(s) and the source, provide a link to the Creative Commons licence, and indicate if changes were made. The images or other third party material in this article are included in the article's Creative Commons licence, unless indicated otherwise in a credit line to the material. If material is not included in the article's Creative Commons licence and your intended use is not permitted by statutory regulation or exceeds the permitted use, you will need to obtain permission directly from the copyright holder. To view a copy of this licence, visit http://creativecommons.org/licenses/by/4.0/ The Creative Commons Public Domain Dedication waiver (http://creativecommons.org/publicdomain/zero/1.0/) applies to the data made available in this article, unless otherwise stated in a credit line to the data. 
Posterior pole retinotomy is a relatively new method of operation. Individual cases as well as small case series have reported similar procedures being used to repair large macular holes [7, 8]. We adopted this surgery in recurrent MHRD because it could be very useful to relieve the reverse vertical traction caused by posterior scleral staphyloma. The procedure is relatively simple with fewer complications, which makes it easier for wide clinical application. The purpose of the present study was to investigate the feasibility and efficacy of posterior pole retinotomy in the treatment of recurrent MRHD in patients with high myopia.

\section{Methods}

\section{Study design}

We performed a retrospective study and analyzed highly myopic patients who received posterior pole retinotomy for their recurrent MHRD at the Department of Ophthalmology in Xiangya Hospital between January 1, 2016 and December 31, 2018. The protocol for this study was approved by the Ethics Committee of Xiangya Hospital, Central South University, Hunan, China. The study adhered to the tenets of the Declaration of Helsinki.

\section{Study participants}

Among highly myopic patients with recurrent MHRD, those who received posterior pole retinotomy were included in our study. These patients all met the following indications for posterior pole retinotomy: (1) axial length $(\mathrm{AL}) \geq 28 \mathrm{~mm}$ with posterior scleral staphyloma; (2) initial MHRD treated with vitrectomy with ILM peeling and silicone oil tamponade; and (3) recurrence of MHRD after silicone oil removal combined with phacoemulsification and intraocular lens (IOL) implantation. In addition, patients with incomplete postoperative and follow-up records were excluded.

\section{Preoperative and postoperative evaluations}

Routine eye examinations, including best-corrected visual acuity (BCVA) measurement, slit lamp examination, fundus examination, intraocular pressure (IOP) measurement by Goldmann applanation tonometry, color fundus photography, A/B-mode ultrasonography, and spectral-domain optical coherence tomography (SDOCT, Carl Zeiss), were performed before and after surgery. Follow-up examinations were performed at 1 week, 1 month, 6 months, 12 months, and 18 months after surgery. The preoperative and postoperative $\mathrm{BCVA}$, axial length (AL, measured by A-mode ultrasonography), and OCT images retrieved from the medical records were analyzed. Data from the last follow-up visit were used to determine the efficacy. SD-OCT images were used to evaluate the anatomical recovery of MHRD. Retinal reattachment was defined when the gap between the retinal neurocortical layer and the retinal pigment epithelium (RPE) layer disappeared.

\section{Surgical technique}

All 11 patients underwent standard transconjunctival 3port 23-gauge PPV by the same ophthalmologist. The operation was performed in the same way for all eyes. After the remaining posterior vitreous body was resected by triamcinolone acetonide $(4 \mathrm{mg} / \mathrm{mL})$-assisted vitrectomy and the epiretinal membrane was removed with forceps, $0.0125 \%$ indocyanine green was used to stain the ILM. Additional ILM peeling was performed when needed to ensure that the peeling area reached the superior and inferior temporal vessel arcades. The arcuate retinotomy was performed at the temporal retina with a distance of $2 \mathrm{PD}$ to the macular hole, with a range extended from $90^{\circ}$ to $120^{\circ}$ after intraocular electrocoagulation. After the retina was flattened with the perfluorocarbon liquid, intraocular laser photocoagulation was performed at the retinal edge of the incision site. Finally, the perfluorocarbon liquid was drained through the gas-liquid exchange and the vitreous cavity was filled with silicone oil. Postoperatively, patients maintained a face-down position for a minimum of $8 \mathrm{~h}$ per day for at least 2 weeks.

At the follow-up visit 1.5 to 3 months after the surgery, silicone oil was extracted from the vitreous cavity through two conventional scleral 23-gauge puncture holes. The gas-liquid exchange was performed repeatedly to remove the residues of emulsified silicone oil in the vitreous cavity. The scleral opening was closed with a 7-0 absorbable suture.

\section{Statistical analysis}

Statistical analysis was performed in SPSS (version 20.0, IBM Corp, Armonk, NY, USA). Visual acuity was converted into the logarithm of the minimal angle of resolution for statistical analysis. Finger counting and hand motion acuities were transformed to 2.0 and 3.0 logarithm of the minimal angle of resolution, respectively. Continuous variables, such as age, AL, follow-up time, and BCVA, are expressed as the mean ( \pm standard deviation). Differences between preoperative and postoperative BCVA and AL were evaluated using paired $t$ test. A $P$ value $<0.05$ was considered as statistically significant.

\section{Results}

\section{General characteristics}

We identified 112 highly myopic patients with recurrent MHRD at our hospital between January 1, 2016 and December 31, 2018. Among them, 11 patients (11 eyes, 4 men and 7 women) received posterior pole retinotomy for their recurrent MHRD. Their baseline clinical characteristics and the history of previous vitrectomies are 
shown in Table 1. Among the 11 eyes, six had one previous vitrectomy and the other five underwent two previous vitreoretinal surgeries. The preoperative and postoperative findings in these patients are shown in Table 2. The mean follow-up time was 13.9 ( \pm 2.4) months (ranged between 12 and 18 months).

\section{Reattachment of detached retina}

After the removal of silicone oil, all 11 eyes achieved anatomical retinal reattachment. There was no retinal detachment occurring during the follow-up period. The retinal reattachment rate was $100 \%$.

\section{Visual acuity outcomes}

The postoperative visual acuities of all eyes were significantly improved compared with the preoperative visual acuities (Table 2). The mean preoperative BCVA LogMAR was 1.87 ( \pm 0.44$)$ for 11 eyes, which improved significantly to $1.15( \pm 0.24)$ at the final visit after surgery $(P<0.05)$.

\section{Macular hole closure}

Postoperative SD-OCT examinations showed that macular holes were completely closed in three eyes (27.3\%) but remained flat open in eight eyes (72.7\%).

\section{Complications}

High IOP occurred in four eyes after surgery (36.4\%). After treatment with IOP-lowering eye drops and removal of silicone oil at 1.5 to 2 months after surgery, high IOPs returned to normal in all patients. No major postoperative complications, such as endophthalmitis, vitreous hemorrhage, or proliferative vitreoretinopathy (PVR), occurred during the follow-up period.

\section{Representative cases}

Case 1 was a 52-year-old woman (Patient 2). Six months ago, she underwent two vitrectomies combined with silicone oil injections due to the myopic macular detachment of the left eye. Fourteen days ago, her vision started to decrease again. Fundus photograph and OCT revealed recurrent MHRD (Fig. 1a, c). After PPV with posterior pole retinotomy and silicon oil tamponade, macular reattachment was achieved and the macular hole was closed (Fig. 1b, d). Postoperative BCVA improved from $\mathrm{FC} / 10 \mathrm{~cm}$ to $20 / 333$ at the final visit 12 months after the surgery.

Case 2 was a 69-year-old woman (Patient 9). Two years ago, she underwent two vitrectomies combined with silicone oil injections for myopic macular detachment of the right eye. This time, she was diagnosed as recurrent MHRD by OCT after presenting with decreased vision for 45 days (Fig. 2). After PPV with posterior pole retinotomy and silicon oil tamponade, macular reattachment was achieved, but the macular hole remained unclosed (Fig. 2f). Postoperative BCVA (Snellen equivalent) improved from 20/500 to 20/200 at the end of the 12-month follow-up period.

\section{Discussion}

MHRD most often occurs in eyes with high myopia [9, 10]. MHRD caused by high myopia accounted for $0.5-$ $5.0 \%$ and $9-21 \%$ of all retinal detachments worldwide and in China, respectively [11]. MHRD is one of the main reasons for visual impairment in highly myopic patients [12]. At the present time, the mechanism of MHRD in high myopia is unclear and involves many factors $[13,14]$, which include axial extension, thickening of the posterior vitreous cortex, abnormal retinal adhesion, long-term vertical and tangential traction of ILM or epiretinal membrane, reverse vertical traction of the

Table 1 Baseline characteristics and clinical history of the study participants

\begin{tabular}{lllllll}
\hline Patient & $\begin{array}{l}\text { Age } \\
\text { (years) }\end{array}$ & Gender & Eye & Days of decreased vision acuity & Times of previous vitrectomy & Methods of previous vitrectomy \\
\hline 1 & $40 \mathrm{~s}$ & male & OD & 7 & 2 & PPV + ILM peeling + silicone oil \\
2 & $50 \mathrm{~s}$ & female & OS & 14 & 2 & PPV + ILM peeling + silicone oil \\
3 & $60 \mathrm{~s}$ & female & OS & 14 & 1 & PPV + ILM peeling + silicone oil \\
4 & $50 \mathrm{~s}$ & male & OD & 7 & 2 & PPV + ILM peeling + silicone oil \\
5 & $50 \mathrm{~s}$ & male & OD & 18 & 1 & PPV + ILM peeling + silicone oil \\
6 & $70 \mathrm{~s}$ & female & OD & 20 & 2 & PPV + ILM peeling + silicone oil \\
7 & $60 \mathrm{~s}$ & female & OS & 7 & 1 & PPV + ILM peeling + silicone oil \\
8 & $50 \mathrm{~s}$ & female & OS & 30 & 1 & PPV + ILM peeling + silicone oil \\
9 & $60 \mathrm{~s}$ & female & OD & 45 & 2 & PPV + ILM peeling + silicone oil \\
10 & $60 \mathrm{~s}$ & male & OD & 16 & 1 & PPV + ILM peeling + silicone oil \\
11 & $60 \mathrm{~s}$ & female & OS & 60 & 1 & PPV + ILM peeling + silicone oil \\
\hline
\end{tabular}


Table 2 Preoperative and postoperative findings in the study participants

\begin{tabular}{|c|c|c|c|c|c|c|c|}
\hline Patient & $\begin{array}{l}\mathrm{AL} \\
(\mathrm{mm})\end{array}$ & $\begin{array}{l}\text { Time of SOR } \\
\text { (months) }\end{array}$ & $\begin{array}{l}\text { Preop BCVA } \\
\text { LogMAR (Snellen } \\
\text { equivalent) }\end{array}$ & $\begin{array}{l}\text { OCT } \\
\text { preop }\end{array}$ & $\begin{array}{l}\text { Post-12 M BCVA } \\
\text { LogMAR (Snellen } \\
\text { equivalent) }\end{array}$ & OCT postop & $\begin{array}{l}\text { Follow-up } \\
\text { (months) }\end{array}$ \\
\hline 1 & 30.48 & 2 & $1.70(20 / 1000)$ & $\begin{array}{l}\mathrm{MD}+\mathrm{MH}+ \\
\mathrm{ERM}\end{array}$ & $1.10(20 / 250)$ & Attached, MH & 12 \\
\hline 2 & 29.22 & 3 & $2.00(\mathrm{FC} / 10 \mathrm{~cm})$ & $\mathrm{MD}+\mathrm{MH}$ & $1.22(20 / 333)$ & $\begin{array}{l}\text { Attached, } \\
\text { closed }\end{array}$ & 18 \\
\hline 3 & 29.40 & 1.5 & $2.00(\mathrm{FC} / 20 \mathrm{~cm})$ & $\mathrm{MD}+\mathrm{MH}$ & $1.10(20 / 250)$ & Attached, MH & 15 \\
\hline 4 & 33.45 & 3 & $3.00(\mathrm{HM} / 20 \mathrm{~cm})$ & $\mathrm{MD}+\mathrm{MH}$ & $1.70(20 / 1000)$ & Attached, MH & 18 \\
\hline 5 & 30.29 & 3 & $1.70(20 / 1000)$ & $\mathrm{MD}+\mathrm{MH}$ & $1.00(20 / 200)$ & Attached, MH & 12 \\
\hline 6 & 30.61 & 3 & $1.70(20 / 1000)$ & $\begin{array}{l}\mathrm{MD}+\mathrm{MH}+ \\
\mathrm{ERM}\end{array}$ & $0.82(20 / 133)$ & $\begin{array}{l}\text { Attached, } \\
\text { closed }\end{array}$ & 12 \\
\hline 7 & 31.79 & 2 & $2.00(\mathrm{FC} / 20 \mathrm{~cm})$ & $\mathrm{MD}+\mathrm{MH}$ & $1.22(20 / 333)$ & Attached, MH & 15 \\
\hline 8 & 30.32 & 3 & $2.00(\mathrm{FC} / 30 \mathrm{~cm})$ & $\mathrm{MD}+\mathrm{MH}$ & $1.10(20 / 250)$ & Attached, MH & 15 \\
\hline 9 & 32.35 & 3 & $1.40(20 / 500)$ & $\begin{array}{l}\mathrm{MD}+\mathrm{MH}+ \\
\mathrm{ERM}\end{array}$ & $1.00(20 / 200)$ & Attached, MH & 12 \\
\hline 10 & 29.48 & 3 & $1.40(20 / 500)$ & $\mathrm{MD}+\mathrm{MH}$ & $1.00(20 / 200)$ & $\begin{array}{l}\text { Attached, } \\
\text { closed }\end{array}$ & 12 \\
\hline 11 & 32.44 & 1.5 & $1.70(20 / 1000)$ & $\mathrm{MD}+\mathrm{MH}$ & $1.40(20 / 500)$ & Attached, MH & 12 \\
\hline
\end{tabular}

ERM macular epiretinal membrane, FC finger count, $H M$ hand move, IOL intraocular lens, MD macular detachment, $M H$ macular hole, Post-12 M 12 months postoperatively; preop, preoperative, SOR silicone oil removal
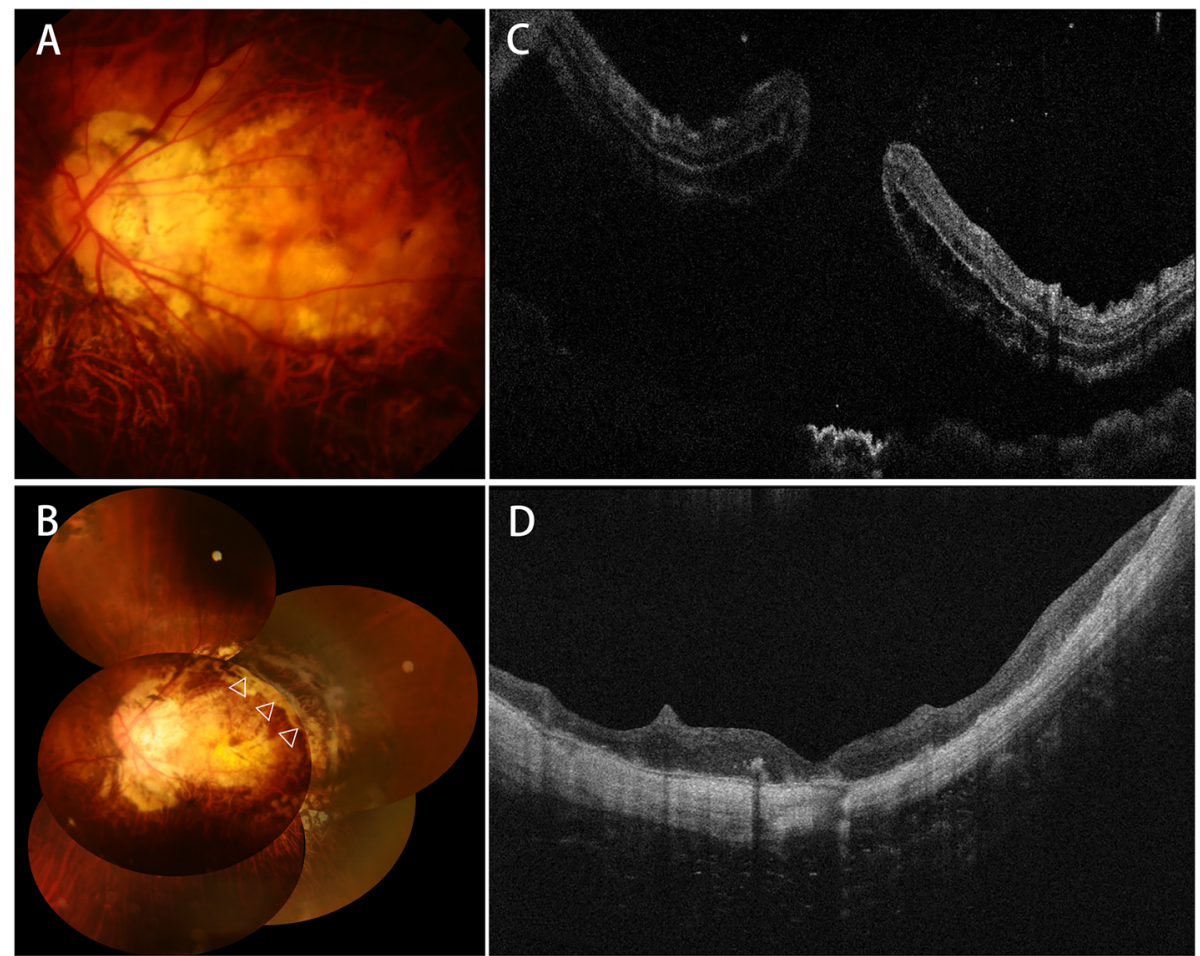

Fig. 1 Color fundus photographs showing macular detachment in the left eye (a), the location (indicated by triangles) of posterior arcuate retinotomy, and retinal reattachment with no proliferative vitreoretiopathy at 6 months after the surgery (b). OCT shows a macular hole with retinal detachment before surgery (c) and macular hole closure and macular reattachment at 3 months after surgery (d) 

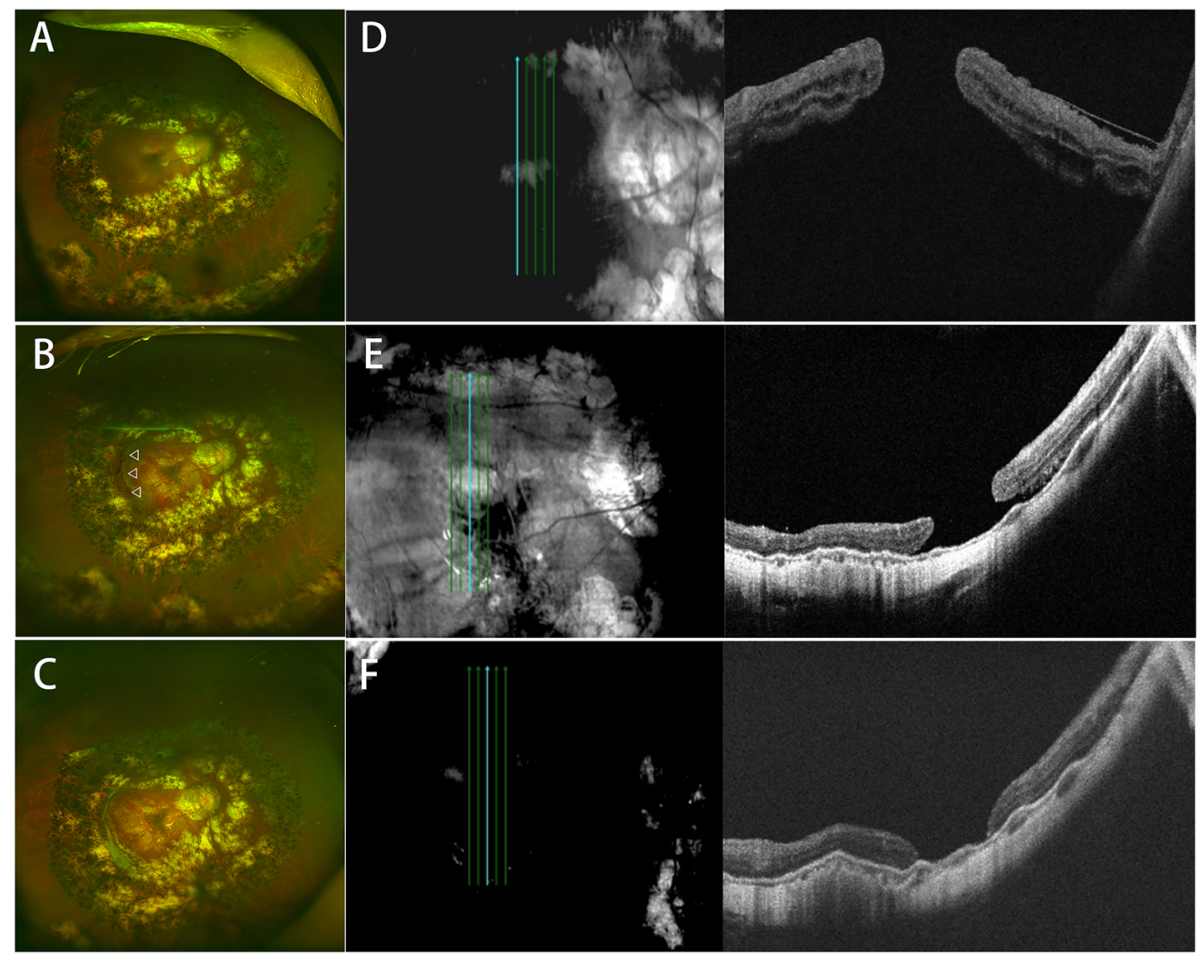

Fig. 2 Color fundus photographs showing posterior pole with retinal detachment in the right eye (a), the location (indicated by triangles) of posterior arcuate retinotomy, retinal reattachment at 1 week after surgery $(\mathbf{b})$, and retinal reattachment with no proliferative membrane at 3 months after surgery (c). OCT shows macular hole, epiretinal membrane, and macular detachment before surgery (d), and macular hole remained unclosed with macular reattachment at 1 week after surgery (e). Macular hole was flat open, and retina remained attached at 3 months following surgery $(\mathbf{f})$

posterior scleral staphyloma, rigid retinal arteriole, and severe choroidal atrophy in the macular area [15-19]. Vitrectomy with ILM peeling and silicone oil tamponade is currently the primary surgical method for treatment of MHRD in patients with high myopia [12, 14]. However, its treatment efficacy is controversial $[2,20]$. Macular hole is difficult to close, and retinal detachment could recur after silicone oil removal or gas absorption [21]. Conventional PPV can clear the posterior vitreous cortex, strip the ILM or epiretinal membrane and remove the tangential or vertical traction factors of the macular area [22]. However, the axial extension and the reverse vertical traction caused by severe posterior scleral staphyloma still cannot be solved. Therefore, the outcomes of repeated PPV in patients with recurrent MHRD are not satisfactory, especially in patients with extremely high axial myopia. Some studies have presented favorable results with PPV combined with temporal scleral shortening, epimacular buckling, and posterior scleral reinforcement [23, 24]. The principle of these surgeries is to reinforce the weak area of the sclera at the posterior pole with special supporting materials in order to reduce the distance between the retina and the sclera [25]. Although this type of surgery has been shown to increase the success of retinal reattachment, it is complex and carries risks of multiple complications, including ischemic injury from the ocular tissue compression (especially the optic nerve or the choroid in the macular area), rejection of the supporting materials, astigmatism, submacular hemorrhage, malposition of buckle, and prolapse of orbital fat [26]. In addition, the exoplant materials used in macular buckle surgery are not commercially available in China, which limits the application of this surgery. It was reported that peripheral retinotomy, which is a common method to treat anterior proliferative vitreoretinopathy, could solve the problem of long axial length and retinal shortening [27]. However, it did not necessarily shorten the distance between the retina and the choroid in the posterior scleral staphyloma due to the convex shape. Moreover, the incision area is too large, which may cause severe damage to the retina and predispose to the postoperative proliferation. Therefore, an effective and safe treatment is still required to treat recurrent MHRD in highly myopic eyes.

In our study, 11 eyes with recurrent MHRD were successfully treated with posterior pole retinotomy. The retinal reattachment was $100 \%$ and no retinal detachment recurred during the follow-up period. Mean BCVA 
improved from $1.87 \pm 0.44$ to $1.15 \pm 0.24$ even though macular holes remained flat open in eight out of 11 eyes after surgery. Four eyes had high IOP postoperatively. No major complications were reported. In recurrent MHRD, since the posterior vitreous cortex, epimacular membrane (if present) and ILM have all been removed during previous operations, traction factors may not be the main reason for surgery failure. Therefore, we assumed the decreased retinal compliance caused by severe posterior scleral staphyloma might be the reason and attempted posterior pole relaxing retinotomy in our case series. Importantly, $45.45 \%$ (5/11) of patients in our study experienced recurrent RD after two vitrectomies combined with silicone oil injections, and they achieved retinal reattachment after posterior pole retinotomy. Though the macular hole closure rate was not high, retinal attachment was achieved in all patients in our study. This surgical approach could relieve the reverse vertical traction caused by the posterior scleral staphyloma and bring the posterior retina closer to the sclera in a simple way (as compared to macular buckling, scleral imbrications, or posterior scleral reinforcement). Our approach did not involve any supporting material, which avoided material-related complications, including astigmatism, ischemia, and rejection.

Posterior pole retinotomy might be a safe and effective surgical method for highly myopic patients with recurrent MHRD after PPV. However, our study had several limitations, including its retrospective design, small sample size, and short follow-up periods. Therefore, future large multi-center randomized controlled trials should be performed to determine the clinical value of posterior pole retinotomy in highly myopic patients with recurrent MHRD. Besides, retinotomy might cause disc changes and compromise retinal functions which needed to be monitored by visual field test, microperimetry and contrast sensitivity tests. High myopic patients already have constricted visual field and compromised visual quality, which makes it difficult to get objective appraisal for the possible damage caused by retinotomy. Considering this, we only recommend posterior pole retinotomy as the last resort for patients with recurrent MHRD after PPV.

\section{Conclusions}

In summary, recurrent macular hole retinal detachment can occur in highly myopic eyes after pars plana vitrectomy. We performed a retrospective study and showed that posterior pole retinotomy is a safe and effective surgery to treat recurrent MHRD after pars plana vitrectomy in highly myopic patients. However, possible functional loss due to retinotomy needs to be monitored in the long term. Indications for posterior pole retinotomy should be carefully determined by surgeons.

\section{Abbreviations}

AL: axial length; BCVA: best-corrected visual acuity; ERM: macular epiretinal membrane; FC: finger count; HM: hand move; IOL: intraocular lens; ILM: internal limiting membrane; IOP: intraocular pressure; MHRD: macular hole retinal detachment; MD: macular detachment; $\mathrm{MH}$ : macular hole; PPV: pars plana vitrectomy; PVR: proliferative vitreoretinopathy; postop: postoperative; preop: preoperative; SD-OCT: spectral-domain optical coherence tomography; SOR: silicone oil removal

\section{Acknowledgements}

We thank LetPub (www.letpub.com) for its linguistic assistance and scientific consultation during the preparation of this manuscript.

\section{Authors' contributions}

All authors contributed to the study conception and design. Material preparation and data collection were performed by XGW, XZZ, and YZ. XGW and $\mathrm{HZX}$ were responsible for the data analysis. The first draft of the manuscript was written by XGW and XZZ. All authors commented on previous versions of the manuscript. HZX revised the manuscript critically for important intellectual content. All authors have read and approved the manuscript in its current state.

\section{Funding}

This work was supported by the National Natural Science Foundation of China (Grant Numbers 81200696). The funders play the roles of collection, analysis of data.

\section{Availability of data and materials}

The datasets used and/or analyzed during the current study are available from the corresponding author on reasonable request.

\section{Declarations}

Ethics approval and consent to participate

The protocol for this study was approved by the Ethics Committee of Xiangya Hospital, Central South University, Hunan, China. All procedures performed in studies involving human participants were in accordance with the ethical standards of the institutional and/or national research committee and with the Helsinki declaration and its later amendments or comparable ethical standards. All participants provided written informed consent before participation.

\section{Consent for publication}

Written informed consent was obtained from both the patients whose cases have been described and for their Color fundus photographs and OCT images for publication of this article. A copy of the written consent is available for review on request.

\section{Competing interests}

The authors declare that they have no competing interests.

Received: 17 March 2021 Accepted: 4 May 2021

Published online: 17 May 2021

\section{References}

1. Takano M, Kishi S. Foveal retinoschisis and retinal detachment in severely myopic eyes with posterior staphyloma. Am J Ophthalmol. 1999;128(4):4726 https://doi.org/10.1016/s0002-9394(99)00186-5.

2. Nakanishi H, Kuriyama S, Saito I, Okada M, Kita M, Kurimoto Y, et al. Prognostic factor analysis in pars plana vitrectomy for retinal detachment attributable to macular hole in high myopia: a multicenter study. Am J Ophthalmol. 2008;146(2):198-204 https://doi.org/10.1016/j.ajo.2008.04.022.

3. Parolini B, Frisina R, Pinackatt S, Gasparotti R, Gatti E, Baldi A, et al. Indications and results of a new l-shaped macular buckle to support a posterior staphyloma in high myopia. Retina. 2015;35(12):2469-82 https:// doi.org/10.1097/IAE.0000000000000613.

4. Zhao X, Ma W, Lian P, Tanumiharjo S, Lin Y, Ding X, et al. Three-year outcomes of macular buckling for macular holes and foveoschisis in highly myopic eyes. Acta Ophthalmol. 2020;98(4):e470-8 https://doi.org/10.1111/a os. 14305 . 
5. Ando Y, Hirakata A, Ohara A, Yokota R, Orihara T, Hirota K, et al. Vitrectomy and scleral imbrication in patients with myopic traction maculopathy and macular hole retinal detachment. Graefes Arch Clin Exp Ophthalmol. 2017; 255(4):673-80 https://doi.org/10.1007/s00417-016-3523-7.

6. Bedda AM, Abdel Hadi AM, Abd Al Shafy MS. A Comparative Study between Vitrectomy with Internal Tamponade and a New Modified Fiber Optic Illuminated Ando Plombe for Cases of Macular Hole Retinal Detachment in Myopic Eyes. J Ophthalmol. 2015;2015:841925. https://doi. org/10.1155/2015/841925-7.

7. Charles S, Randolph JC, Neekhra A, Salisbury CD, Littlejohn N, Calzada JI. Arcuate retinotomy for the repair of large macular holes. Ophthalmic Surg Lasers Imaging Retina. 2013;44(1):69-72 https://doi.org/10.3928/23258160-2 0121221-15.

8. Karacorlu M, Sayman Muslubas I, Hocaoglu M, Arf S, Ersoz MG. Double arcuate relaxing retinotomy for a large macular hole. Retin Cases Brief Rep. 2019;13(2):167-70 https://doi.org/10.1097//CB.0000000000000551.

9. Alkabes M, Pichi F, Nucci P, Massaro D, Dutra Medeiros M, Corcostegui B, et al. Anatomical and visual outcomes in high myopic macular hole (HM$\mathrm{MH})$ without retinal detachment: a review. Graefes Arch Clin Exp Ophthalmol. 2014;252(2):191-9 https://doi.org/10.1007/s00417-013-2555-5.

10. De Giacinto C, Pastore MR, Cirigliano G, Tognetto D. Macular hole in myopic eyes: a narrative review of the current surgical techniques. J Ophthalmol. 2019;2019:3230695. https://doi.org/10.1155/2019/3230695-10.

11. Zheng $Y$, Kang $M$, Wang $H$, Liu H, Sun T, Sun $X$, et al. Inverted internal limiting membrane insertion combined with air tamponade in the treatment of macular hole retinal detachment in high myopia: study protocol for a randomized controlled clinical trial. Trials. 2018;19(1):469 https://doi.org/10.1186/s13063-018-2833-y.

12. Ortisi $E$, Avitabile T, Bonfiglio V. Surgical management of retinal detachment because of macular hole in highly myopic eyes. Retina. 2012;32(9):1704-18 https://doi.org/10.1097/IAE.0b013e31826b671c.

13. Lai CC, Chen YP, Wang NK, Chuang LH, Liu L, Chen KJ, et al. Vitrectomy with internal limiting membrane repositioning and autologous blood for macular hole retinal detachment in highly myopic eyes. Ophthalmology. 2015;122(9):1889-98 https://doi.org/10.1016/j.ophtha.2015.05.040.

14. Nishimura A, Kimura M, Saito Y, Sugiyama K. Efficacy of primary silicone oil tamponade for the treatment of retinal detachment caused by macular hole in high myopia. Am J Ophthalmol. 2011;151(1):148-55 https://doi.org/1 0.1016/j.ajo.2010.07.023.

15. Song $M$, Shen $M$, Zhou $Y$, Zheng $K$, Zhai $Y$, Xiao M, et al. Observation of vitreous features using enhanced vitreous imaging optical coherence tomography in highly myopic retinoschisis. Retina. 2019;39(9):1732-41 https://doi.org/10.1097/IAE.0000000000002226.

16. Gupta P, Saw SM, Cheung CY, Girard MJ, Mari JM, Bhargava M, et al. Choroidal thickness and high myopia: a case-control study of young Chinese men in Singapore. Acta Ophthalmol. 2015;93(7):e585-92 https://doi. org/10.1111/aos.12631

17. Li T, Wang X, Zhou Y, Feng T, Xiao M, Wang F, et al. Paravascular abnormalities observed by spectral domain optical coherence tomography are risk factors for retinoschisis in eyes with high myopia. Acta Ophthalmol. 2018;96(4):e515-23 https://doi.org/10.1111/aos.13628.

18. Shinohara K, Shimada N, Takase H, Ohno-Matsui K. Functional and structural outcomes after fovea-sparing internal limiting membrane peeling for myopic macular retinoschisis by microperimetry. Retina. 2020;40(8):1500-11 https://doi.org/10.1097/IAE.0000000000002627.

19. Ohno-Matsui K, Jonas JB. Posterior staphyloma in pathologic myopia. Prog Retin Eye Res. 2019;70:99-109 https://doi.org/10.1016/j.preteyeres.2018.12.001

20. Ho TC, Ho A, Chen MS. Vitrectomy with a modified temporal inverted limiting membrane flap to reconstruct the foveolar architecture for macular hole retinal detachment in highly myopic eyes. Acta Ophthalmol. 2018; 96(1):e46-53 https://doi.org/10.1111/aos.13514.

21. Chen X, Yao Y, Hao X, Liu X, Liu T. A comparative study of Vitrectomy combined with internal limiting membrane peeling for the treatment of idiopathic macular hole with air or C3F8 intraocular Tamponade. J Ophthalmol. 2018;2018:1672501.https://doi.org/10.1155/2018/1672501-6.

22. Laviers H, Li JO, Grabowska A, Charles SJ, Charteris D, Haynes RJ, et al. The management of macular hole retinal detachment and macular retinoschisis in pathological myopia; a UK collaborative study. Eye (Lond). 2018:32(11): 1743-51 https://doi.org/10.1038/s41433-018-0166-4.
23. Ma J, Li H, Ding X, Tanumiharjo S, Lu L. Effectiveness of combined macular buckle under direct vision and vitrectomy with ILM peeling in refractory macular hole retinal detachment with extreme high axial myopia: a 24month comparative study. Br J Ophthalmol. 2017;101(10):1386-94 https:// doi.org/10.1136/bjophthalmol-2016-310123.

24. Cao K, Wang J, Zhang J, Yusufu M, Jin S, Zhu G, et al. The effectiveness and safety of posterior scleral reinforcement with vitrectomy for myopic foveoschisis treatment: a systematic review and meta-analysis. Graefes Arch Clin Exp Ophthalmol. 2020;258(2):257-71 https://doi.org/10.1007/s00417-01 9-04550-5.

25. Xue A, Zheng L, Tan G, Wu S, Wu Y, Cheng L, et al. Genipin-Crosslinked donor sclera for posterior scleral contraction/reinforcement to fight progressive myopia. Invest Ophthalmol Vis Sci. 2018;59(8):3564-73 https:// doi.org/10.1167/iovs.17-23707.

26. Susvar P, Sood G. Current concepts of macular buckle in myopic traction maculopathy. Indian J Ophthalmol. 2018;66(12):1772-84 https://doi.org/1 0.4103/ijo.IJO_1126_18.

27. Bovey EH, De Ancos E, Gonvers M. Retinotomies of 180 degrees or more. Retina. 1995;15(5):394-8. https://doi.org/10.1097/00006982-199515050-00004.

\section{Publisher's Note}

Springer Nature remains neutral with regard to jurisdictional claims in published maps and institutional affiliations.
Ready to submit your research? Choose BMC and benefit from:

- fast, convenient online submission

- thorough peer review by experienced researchers in your field

- rapid publication on acceptance

- support for research data, including large and complex data types

- gold Open Access which fosters wider collaboration and increased citations

- maximum visibility for your research: over $100 \mathrm{M}$ website views per year

At BMC, research is always in progress.

Learn more biomedcentral.com/submissions 